\title{
Vehicle Routing Problem with Deliveries and Pickups: Modelling Issues and Meta-heuristics Solution Approaches
}

\author{
Niaz A. Wassan ${ }^{1}$ and Gábor Nagy ${ }^{2}$ \\ ${ }^{1,2}$ Kent Business School, Centre for Logistics \& Heuristic Optimisation (CHLO) \\ University of Kent, Canterbury, $U K$ \\ ${ }^{1}$ N.A.Wassan@kent.ac.uk, ${ }^{2}$ G.Nagy@kent.ac.uk
}

\begin{abstract}
The paper investigates a class of extensions to the vehicle routing problem. Different problem versions - some well-known, some more recent - are explained and placed in a taxonomy. A central focus of the paper is on the assumptions generally made in the literature and on the benefits of not making too restrictive assumptions. Research issues on novel problem classes are highlighted. An Integer Linear Programming (ILP) formulation is also presented. It is also shown how this formulation can be adapted to cater for other problem versions. This paper also discusses various solution methodologies including meta-heuristics to solve the models and what more is needed the vehicle routing problem.
\end{abstract}

Keywords: Logistics, Transportation, Vehicle routing, Metaheuristics, Backhauling, Optimization

\section{Introduction}

In the Vehicle Routing Problem (VRP), we are given a set of customers with known demands, a set of vehicles (typically homogeneous) and a depot. The problem is to design least-cost vehicle routes originating and terminating at the depot to service customers, subject to vehicle capacity constraints and in some cases there may be route length constraints. The vehicle routing problem with deliveries and pickups (VRPDP) extends the VRP by having goods transported from a depot to customers but also from customers to the depot. The VRPDP is an important logistics problem with wide applications, mainly in reverse logistics. (On one hand, an increasingly environmentally-conscious population means more collection of recyclable goods. On the other hand, in the current grave economic situation, companies are ever more mindful of the savings that can be made by combining deliveries and pickups.) At the same time, it is a challenging combinatorial optimisation problem, containing complex constraints not present in other vehicle routing problems. (All the versions of the VRPDP discussed here are, by virtue of being extensions to the travelling salesman problem, NP-hard.) The reader's attention is drawn to the excellent reviews on this subject by Toth and Vigo (2002), Berbeglia, Cordeau, Gribkovskaia and Laporte (2007) and Parragh, Doerner and Hartl (2008).

The motivation for this paper is the emergence of novel areas of VRP with deliveries and pickups. These areas model flexibility and practical assumptions as required by the logistics companies in reality. Moreover, while there is a burgeoning literature on the VRPDP, there is also a lot of confusion in the minds of VRP researchers not specialising in this field about its various versions. We felt motivated to write this paper having been to numerous conferences and finding that many colleagues were unaware of some newer VRPDP topics and confused about the differences between VRPDP versions. Our aim is to give a concise overview of the VRPDP and a detailed explanation of some new research areas. It is not our aim to give details of more traditional versions, as there exist already some excellent literature reviews (Berbeglia, Cordeau, Gribkovskaia and Laporte 
(2007) and Parragh, Doerner and Hartl (2008)). In line with our aim the paper is problem-focused rather than solution-focused; modelling issues are at the heart of this work. We hope it will serve as a good introduction to the VRPDP for VRP researchers moving into this topic and especially for PhD students who may consider the VRPDDP as their research topic.

In the next section, we present a traditional view of the VRPDP, including commonly made assumptions underpinning these classical VRPDP models. Sections 3 to 5 challenge some classical assumptions and explain what happens if we set them aside. Section 6 presents a mathematical programming formulation that is sufficiently versatile to cover various models in the VRPDP together with some numerical experiments. Section 7 gives an overview of metaheuristic methodologies, while the last section presents our conclusions.

\section{Models within the VRPDP}

We prefer the expression VRP with deliveries and pickups (rather than pickups and deliveries) to signify that all goods either come from the depot, or arrive there. There is no inter-customer transportation of goods. We do not look in this paper at pickup-anddelivery problems, where goods are taken directly from one customer to another. ("Goods" may be passengers, as in the well-known dial-a-ride problem.) While this is an important problem, it is not properly an extension to the VRP, as it lacks the multi-stop tour planning aspect. Customers that receive goods from the depot are known as linehauls or deliveries; customers who send goods are backhauls or pickups. It is perfectly possible that a customer may wish to both send and receive goods. This case is known as combined demands, if all customers are either pure deliveries or pure pickups it is referred to as single demands.

Since the seminal paper of Casco, Golden and Wasil (1988), initially most VRPDP research focused on three important problem classes (see also Figure 1):

(i) The VRP with Backhauling (VRPB) is a special case of single demands, built on the premise that all deliveries must be served before pickups can begin. (We explain the motivation for this in some detail later on.)

(ii) The VRP with Mixed Deliveries and Pickups (VRPMDP), on the other hand, does not make the above assumption and allows deliveries and pickups to occur in any order on a vehicle route.

(iii) The VRP with Simultaneous Deliveries and Pickups (VRPSDP) is a case of combined demands where the vehicle makes a single stop to both deliver and pick up goods. However, this expression was also used to denote that customers simultaneously have delivery and pickup demand, i.e. as a synonym for combined demands.

It is worthwhile to note here that the VRPMDP may be modelled as a VRPSDP by adding a pickup of zero to each linehaul and a delivery of zero to each backhaul. This does not make the model unduly more complicated. However, the VRPSDP cannot be modelled as a VRPMDP. One can create two fictitious co-located customers (one purely linehaul one purely backhaul) to convert the problem from combined demands to single demands, but if this was solved as a VRPMDP the requirement of a simultaneous visit may not be satisfied. 


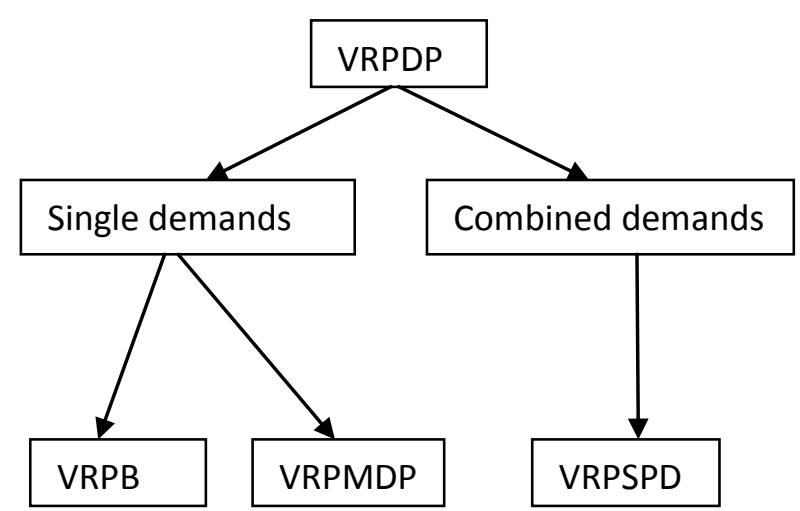

Figure 1. The Traditional Classification of VRPDP Models

In this paper, we investigate the two assumptions referred to above, namely:

(a) For single demands, all deliveries must be completed before pickups may begin.

(b) For combined demands, delivery and pickup must take place at the same time.

Challenging these assumptions gives rise to exciting new problem classes, which will be explained in Sections 3, 4 and 5.

\section{The Vehicle Routing Problem with Restricted Mixing of Deliveries and Pickups}

For any Operational Research problem, the more restrictions one has, the smaller the feasible region. Unnecessary assumptions may mean that the optimum is no longer seen as feasible, leading to suboptimal solutions. Hence, an Operational Research analyst must always investigate every assumption to see whether it is really required. What, then, is the justification for requiring that all deliveries must be completed before pickups may begin? Let us look at the case where deliveries and pickups may occur in any order (known as the VRPMDP). In this case, it is possible that, after a number of stops, the goods picked up obstruct access to the delivery goods. The driver has no alternative but to remove pickup goods to access the delivery, then put these back on the vehicle. If this happens often, the driver will be very angry with the Operational Researcher who designed the route, even if it is the optimal route with respect to total distance! This issue is known as the load shuffling problem, and is the main practical argument for the VRPB assumption. Moreover, we observe that the VRPMDP is harder to solve than the VRPB. This is because in the VRPB the load carried on a vehicle decreases at every stop until the vehicle is empty; afterwards it increases. Hence, to check the maximum capacity requirement it is sufficient to check that the total delivery load and the total pickup load are both less than or equal to the capacity of the vehicle. On the other hand, in the VRPMDP, one has a fluctuating load, as at each customer the load may go up or down. This means that feasibility has to be checked at very arc of the vehicle tour. However, this merely requires the analyst to work harder and is not a genuine obstacle to using the VRPMDP! (Finally, we note that most VRPB papers also assume that there is a fixed fleet of which every vehicle must be used and that no route may contain only backhauls. The first of these is a logical practical requirement that may be present for any VRP problem; however, for some reason, it is often used in the VRPB but not in the VRPMDP. We cannot see any practical justification for the second assumption.) Thus, we can return to the load shuffling problem and look at three examples of modelling and dealing with this issue.

Hoff and Løkketangen (2006) describe the distribution strategy of a Norwegian mineral water distributor. To eliminate the need for removing pickup goods on board to access delivery goods, in the first part of the vehicle tour they perform deliveries only so that a 
clear channel in the middle of the vehicle is created to reach all the way to the far end. Then, both deliveries and pickups are performed such that pickups are taken through the empty channel to the end of the vehicle. In the final part of the tour pickups only are performed, filling the available empty spaces. (See Figure 2a.) This strategy is applicable to problems where the goods are small enough for a corridor to be created.

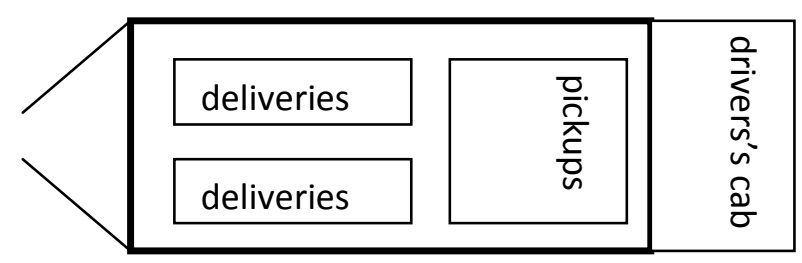

(a)

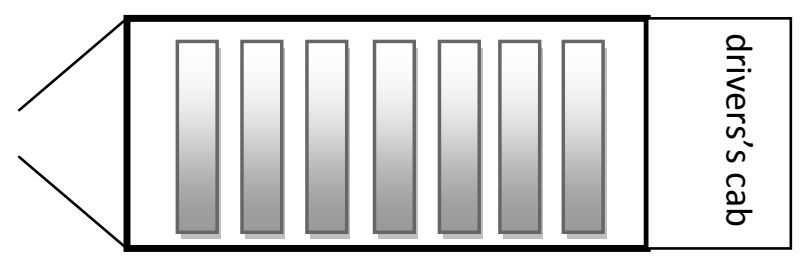

(b)

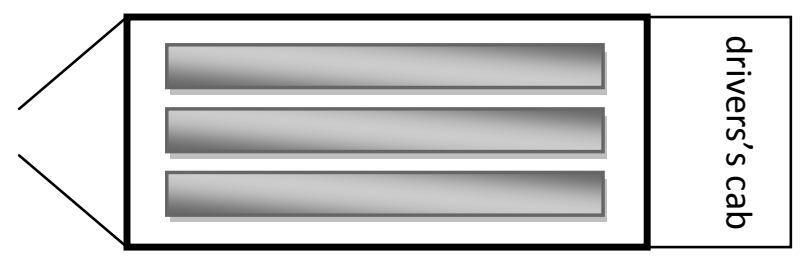

(c)

Figure 2. Illustrations of Vehicle Designs

On the other hand, in the problem considered by Battarra, Erdoğan, Laporte and Vigo (2010), goods fill the width of the vehicle so that only the item nearest the vehicle door is directly accessible. (See Figure $2 b$.) They refer to an example of deliveries and collecting bicycles, which are stored on a linear rack within the vehicle. Clearly, frequent unloading and reloading of goods may be required and one of the aims is to minimise the number of such operations called handling costs. The authors investigate a number of handling policies: (1) placing pickups near the door of the vehicle, (2) placing pickups at the back, and (3) choosing at each customer between the first and second policies. In the last case, this choice is incorporated in the problem formulation so that the optimiser can make the choice. As an alternative to the above, first the routing problem may be solved exactly and then the problem of loading/unloading may be solved exactly. (Of course as the two aspects are optimised separately this is a heuristic rather than an exact method.) The authors found that the third policy and the two-phase method are equally good.

Nagy, Wassan and Salhi (2013) give the following small example, similar to that of Hoff and Løkketangen (2006). The vehicle can carry three items only, and they can fit parallel to each other, filling the length of the vehicle. (See Figure 2c.) As the door is narrower than the full width of the vehicle, if there are three items onboard, it is necessary to remove the middle item to access the ones on the side. The authors investigate the VRPB and VRPMDP policies, and compare it to the following compromise policy: three items are only allowed onboard if they are all deliveries or all pickups. (This policy eliminates to need for unloading and reloading.) 
Apart from the above approaches, an interesting way to model the load shuffling problem would be by considering the exact dimensions of the goods onboard. This would lead to combining the VRPDP with the (two- or three-dimensional) bin-packing or strippacking problem. Such a model does not yet exist in the literature, although recently there is a significant growth of interest in so-called routing problems with loading constraints. (We note that there are several papers on pickup-and-delivery problems with loading constraints.) For an overview of such problems, see the review of Iori and Martello (2010).

As the reader can see from the first and third examples on the load shuffling problem, having some free space on board can be beneficial. This observation led to the development of a novel research area with the VRPDP, the VRP with Restricted Mixing of Deliveries and Pickups (VRPRMDP). In this model, delivery and pickup goods may be together on a vehicle, but only if a certain amount of free space is also present. (The vehicle can be full to capacity if it contains only delivery or only pickup goods.) The most common version of this model stipulates that when both kinds of goods are present onboard, a given percentage of the vehicle must be empty (to form a corridor facilitating access to goods). A 20-25\% free space is commonly viewed as an acceptable figure.

We note that both the VRPB and the VRPMDP are special cases of the VRPRMDP. The VRPB is equivalent to requiring that all of the vehicle must be empty if linehaul and backhaul goods are both present - meaning that these goods cannot be taken together, further implying that all deliveries must be completed before pickups may begin. The VRPMDP is equivalent to saying that a free space of zero is needed, i.e., there is no restriction on the order deliveries and pickups are performed.

A central research issue in the VRPRMDP is the influence of the restriction parameter on the solution quality. It is clear that the same instance, solved under the VRPB assumption, will have a worse solution quality than if the VRPMDP assumption is used: Reimann and Ulrich (2006) found an 11\% average solution gap on their instances (which contained also a time windows restriction), while Nagy, Wassan and Salhi (2013) found a $16 \%$ gap using their unified heuristic and a $10 \%$ gap when referring to best-known solutions. The latter also note that the gap between VRPB and VRPMDP increases with the proportion of backhauls (until there are an equal number of linehauls and backhauls) and that then presence of a maximum time criterion or time windows constraints decrease this gap. The main observation of Nagy, Wassan and Salhi (2013) is that the solution quality is not a linear, but a concave function of the free space parameter. This means, on one hand, that by allowing linehaul and backhaul goods both to be present on a nearly empty vehicle already gives a drastic reduction in route length from the VRPB. On the other hand, if a small corridor is sufficient to overcome the load shuffling problem, then the VRPRMDP solution is only slightly worse than the VRPMDP solution.

There are very few papers on the VRPRMDP. Casco, Golden and Wasil (1988) introduced the problem together with the VRPB and the VRPMDP. Wade and Salhi (2002) proposed a constructive heuristic. Reimann and Ulrich (2006) designed an ant colony metaheuristic and allowed for longer service stops if rearrangement of goods is required. Tütüncü, Carreto and Baker (2009) used a GRASP metaheuristic. Nagy, Wassan and Salhi (2013) applied a reactive tabu search metaheuristic and carried out a detailed study of the effect of the free space parameter on the solution quality.

\section{The Vehicle Routing Problem with Divisible Deliveries and Pickups}

Clearly, it is convenient for customers to have their delivery and pickup needs satisfied in a single visit. What are the benefits of allowing more than one visit? Due to the fluctuating load on the vehicle in many VRPDP versions, a route may not be feasible even if its total delivery and total pickup loads are both below its maximum capacity. There are a number of ways to make such a route feasible. For example, Mosheiov (1994) suggested 
that feasibility may be achieved by reinserting the depot onto a different solution on the vehicle tour. Another idea - and the focus of this section - is to consider serving some of the customers twice. Nagy, Wassan, Speranza and Archetti (2012) present the following example. Let there be three customers placed on a straight line at distances 1, 2 and 3 from the depot. Customer 1 has delivery of 1 and pickup of 3, customer 2 has delivery and pickup both of 2, customer 3 has delivery of 3 and pickup of 1; vehicles have a capacity of 3. The optimal solution under the one-visit-per-customer assumption has three vehicles, each visiting a single customer; total route length is 12 . However, by allowing the delivery and pickup of customer 2 to be served separately, we arrive at an optimal solution with two vehicles: one delivers to customer 2 then visits customer 1 , the other visits customer 3 then picks up from customer 2; total route length is 10 . Thus, allowing more than one visit may reduce the total route length and the number of vehicles. It is possible to reduce route length even for a single vehicle, as can be shown by the example given by Hoff and Løkketangen (2006). The depot is located at $(0,1)$. There are four customers located at $(1,1),(1,2),(4,2)$ and $(4,0)$. Their delivery and pickup values are $(2,4),(2,4),(6,1)$ and $(1,2)$, respectively. The vehicle has a capacity of 11 . If only one visit is allowed, customers should be visited in the order 3-4-1-2, with a route length of 11.70. If this assumption is not made, then a drop should first be made at customer 1 , then customers 2,3 and 4 should be served, finally a pickup should be made from customer 1. This route has length 11.16.

Clearly, the issue of splitting (i.e. serving customers twice) only arises if customers have combined demands. This situation traditionally was equivalent to the VRPSDP. If deliveries and pickups can be served separately, the new model is named the VRP with Divisible Deliveries and Pickups (VRPDDP). It should be noted that the VRPDDP may be modelled, at the expense of doubling the number of customers, as a VRPMDP. (Each genuine customer is replaced by two fictitious ones, one purely delivery, the other purely pickup.) Moreover, by adding zero delivery/pickup values, the VRPMDP may further be modelled as a VRPSDP. Doubling the number of the customers, of course, makes the problem harder to solve.

There are obvious similarities between the VRPDDP and the Split Delivery VRP (SDVRP), as both are based on the premise of allowing more than one visit to a customer. However, in the VRPDDP usually only two visits are allowed, and deliveries and pickups may not themselves be split. For a review on the SDVRP, the reader is referred to Archetti and Speranza (2012). Some of the theoretical properties of the SDVRP are also valid in the VRPDDP, but notably some are not. For example, in the SDVRP there is always an optimal solution such that each pair of routes contains no more than one customer in common. This is not true for the VRPDDP. For a more detailed discussion on these issues, see Nagy, Wassan, Speranza and Archetti (2012).

While the VRPDDP only specifies that up to two visits may be made to a customer, some models consider a more specific solution structure. In a so-called lasso solution, vehicles first visit some customers for delivery only. Then, another set of customers are visited and their delivery and pickup needs are served simultaneously. Finally, the vehicle picks up from the first set of customers, following the reverse of the outbound route. (It is assumed that the delivery and the pickup of every customer are served by the same vehicle.) This model is in line with the pattern used in practice by many logistics companies, see e.g. Halskau, Gribkovskaia and Myklebost (2001). By not picking up from the first set of customers on the outbound leg, the vehicle will be relatively lightly loaded in the second leg, thus alleviating the planning problems caused by the fluctuating vehicle load. Hoff and Løkketangen (2006) note that such a structure also alleviates the load shuffling problem, as on the leg of the route where deliveries and pickups are performed simultaneously, there is sufficient free space to facilitate rearrangement of goods on-board. Moreover, it is easier to construct a lasso solution than one without such a general structure. Often, a lasso VRPDDP solution can be constructed from an 
infeasible VRPSDP solution. We note that while lasso route structures are common, they may not always be the best. The experiments of Nagy, Wassan, Speranza and Archetti (2012) found few lasso solutions, as customers often ended up being split across two vehicles. For a more detailed discussion on lasso and other route shapes, see Gribkovskaia, Halskau, Laporte and Vlček (2007).

Clearly, for the same instance, not requiring a simultaneous visit for delivery or pickup will give at least as good a solution as the VRPSDP one. The following interesting question arises: what is the maximum improvement one may achieve by removing the simultaneous visit restriction? This was answered by Nagy, Wassan, Speranza and Archetti (2012), who proved that the total route length may be decreased by $50 \%$, but no more. Similarly, the number of vehicles required in the VRPDDP may be as little as half of that in the VRPSDP. In practice, one should not expect the difference between the VRPDDP and the VRPSDP solutions to be so dramatic. Numerical experiments carried out by Nagy, Wassan, Speranza and Archetti (2012) found improvements of up to $16 \%$. Another measure that may be used instead of route length is the concept of detour costs, i.e. the difference between the VRPSDP/VRPDDP solutions and the solution to the VRP which results when pickups are removed. Nagy, Wassan, Speranza and Archetti (2012) has shown that the detour cost of a VRPDDP may be up to 100\% less than the detour cost of a VRPSDP; in practice, even average savings in detour cost may be as large as $44 \%$.

The literature on the VRPDDP is also scarce. Most papers focus on the single-vehicle case, the Travelling Salesman Problem with Divisible Deliveries and Pickups (TSPDDP); we begin with these. Mosheiov (1994) introduced the Reinsert heuristic, where feasibility can be achieved by reinserting the depot to a different position on the tour. Anily (1996), somewhat oddly, made the deliveries-before-pickups assumption, hence forcing all but one customer to be served twice. Halskau, Gribkovskaia and Myklebost (2001) introduced the lasso solution concept and proposed a constructive heuristic. (They noted that the VRPDDP may be solved using a cluster-first, TSPDDP-second approach.) Mitra (2005, 2008) allows both deliveries and pickups to be split themselves into several visits. A simple constructive heuristic and a cluster-based heuristic are presented, respectively. Gribkovskaia, Halskau, Laporte and Vlček (2007) presented a constructive heuristic and a tabu search metaheuristic based on splitting and merging customers. The main focus of this paper is on route shapes. Gribkovskaia, Laporte and Shyshou (2008) extend the above methods to the TSP with Deliveries and Selective Pickups, where some pickups may be omitted (for a penalty). The VRPDDP itself was introduced by Salhi and Nagy (1999) and Nagy and Salhi (2005). Their constructive heuristics were based on splitting and merging customers. Nagy, Wassan, Speranza and Archetti (2012) designed a reactive tabu search metaheuristic and investigated (a) the types of instances where there are large cost differences between the VRPSDP and the VRPDDP models, (b) the characteristics of customers that are served twice in the VRPDDP, and (c) the shapes of VRPDDP routes.

\section{The Vehicle Routing Problem with Restricted Mixing of Divisible Deliveries and Pickups}

In the previous two sections we looked at relaxing two assumptions in the VRPDP, leading to two new models, namely the VRPRMDP and the VRPDDP. It is interesting to consider whether these aspects may be combined. Hoff and Løkketangen (2006) introduced this new model, namely the VRP with Restricted Mixing of Divisible Deliveries and Pickups (VRPRMDDP). In their model, customers have combined demands, and there is a requirement of free space when both deliveries and pickups are present. Let us show this situation by an example. Let there be three customers on a straight line, at distances 1, 2 and 3 from the depot. Their delivery and pickup values are $(1,1),(2,1)$ and $(1,2)$, respectively. The vehicle has a capacity of 4 and there is a requirement for $25 \%$ free space when both linehaul and backhaul goods are present. The 
optimal solution without the free space requirement has length 6 , an optimal route is $0-1-$ $2-3-0$. In the presence of this requirement, and the assumption that only one visit may be made to the customer, the route length increases to 8 , an optimal route is $0-2-1-3-0$. If splitting is allowed, then an optimal solution of length 6 may again be found, first delivering only to customer 1 , then visiting customers 2 and 3, finally picking up from customer 1 .

There are only two papers addressing this problem. Hoff and Løkketangen (2006) solve the TSPRMDDP using a lasso solution. A free space of $20 \%$ is specified, although some other values were investigated. A 2-opt improvement heuristic embedded in tabu search is used. Hoff, Gribkovskaia, Laporte and Løkketangen (2009) extend the above to the VRPRMDDP, again using tabu search. Different values of the free space are investigated. The authors also compare lasso solutions against not having a fixed route shape. They note that while specifying a lasso structure reduces the feasible set and thus potentially misses the optimal solution, the general shape heuristic requires a doubling of the customers and is hence slower. Using the same number of iterations, a lasso heuristic will often give better solutions than the one based on doubling customers.

At the end of this section, as a summary, we present a diagram of old and new VRPDP models in Figure 3.

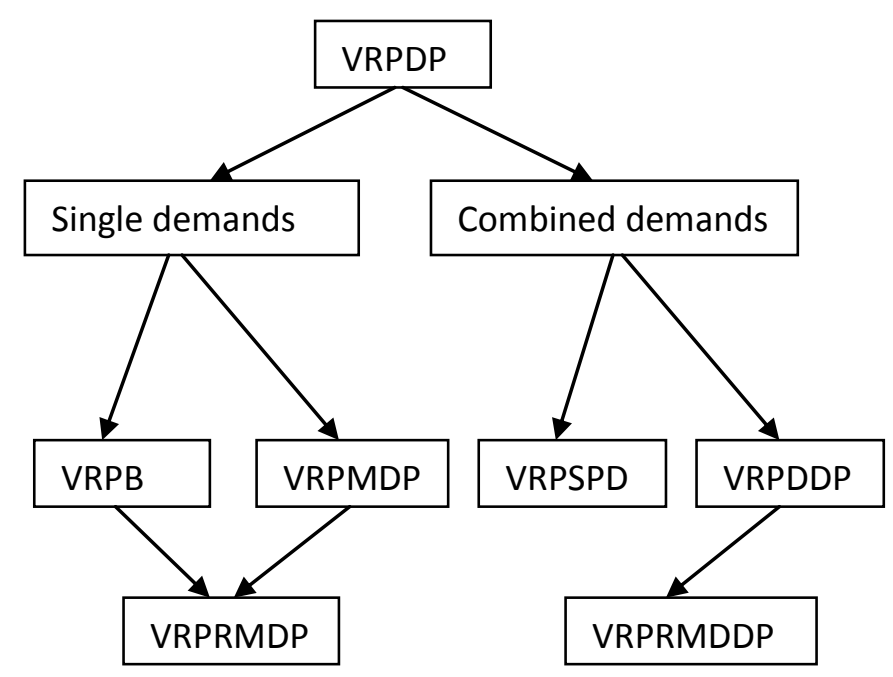

Figure 3. A Classification of VRPDP Models including Novel Problem Classes

\section{Integer Linear Programming (ILP) models for VRPDP}

We first present a model for the VRPSDP, then show how it may be modified to model the other problem classes. Let us introduce the following notation.

Input variables

$d_{i j} \quad$ the distance between locations $i$ and $j$

$q_{i} \quad$ the delivery demand of customer $i$

$b_{i} \quad$ the backhaul (pickup) demand of customer $i$

C vehicle capacity

\section{Decision variables}

$x_{i j} \quad$ equals 1 if there is a vehicle travelling from location $i$ to location $j$; equals 0 otherwise

$R_{i j} \quad$ the amount of delivery goods on board on arc $i j$

$P_{i j} \quad$ the amount of pickup goods on board on arc $i j$

The VRPSDP can be modelled as follows. 
Minimise $\quad Z=\sum_{i=0}^{n} \sum_{j=0}^{n} d_{i j} x_{i j}$

(1)

subject to $\quad \sum_{i=0}^{n} x_{i j}=1$

$j \in\{1, \ldots, n\}$

(2)

$\sum_{i=0}^{n} x_{j i}=1$

$j \in\{1, \ldots, n\}$

(3)

$\sum_{i=0}^{n} R_{i j}-q_{j}=\sum_{i=0}^{n} R_{j i} \quad j \in\{1, \ldots, n\}$

(4)

$\sum_{i=0}^{n} P_{i j}+b_{j}=\sum_{i=0}^{n} P_{j i} \quad j \in\{1, \ldots, n\}$

(5)

$\sum_{i=1}^{n} P_{0 i}=0$

(6)

$\sum_{i=1}^{n} R_{i 0}=0$

(7)

$$
\begin{array}{rrrr}
R_{i j}+P_{i j} & \leq C x_{i j} & i \in\{0, \ldots, n\}, j \in\{0, \ldots, n\} \\
x_{i j} & \in\{0,1\} & i \in\{0, \ldots, n\}, j \in\{0, \ldots, n\}
\end{array}
$$

(9)

$$
R_{i j} \quad \geq 0
$$$$
i \in\{0, \ldots, n\}, j \in\{0, \ldots, n\}
$$

(10)

$$
P_{i j} \geq 0 \quad i \in\{0, \ldots, n\}, j \in\{0, \ldots, n\}
$$

(11)

We present below a brief line-by-line explanation for this formulation.

(1) The objective is to minimise the total distance travelled by the vehicles.

(2)-(3) Every customer is served exactly once.

(4)-(5) Flow conservation constraints. (These constraints also eliminate subtours.)

(6)-(7) Vehicles start with zero pickup load and finish with zero delivery load.

(8) Maximum capacity constraint.

(10)-(11) Set $x_{i j}$ as zero-one and $R_{i j} / P_{i j}$ as non-negative variables.

The above formulation is based on a two-index VRP formulation. This is achieved by not identifying the vehicle itself as this can be derived from the result. If the problem stipulates that a given number of vehicles must be used, we add the constraint:

$$
\sum_{i=1}^{n} x_{0 i}=K
$$

We can modify the above VRPSDP formulation to cater for other VRPDP versions. 
(a) The VRPMDP can be modelled as a VRPSDP by adding zero pickup values to all delivery customers and zero delivery values to all backhauls.

(b) The "restricted mixing" assumption necessitates an additional constraint on capacity on arcs that carry a mixture of delivery and pickup goods. Nagy, Wassan and Salhi (2013) have shown that it is sufficient instead to place this restriction only on arcs that go from a backhaul to a linehaul. Let $\gamma$ be the percentage of free space required when a mixture of goods is onboard and assume that the first $m$ customers are linehauls and the rest backhauls. Then, the following constraint models our restriction:

$$
R_{i j}+P_{i j} \leq(1-\gamma) C x_{i j} i \in\{m+1, \ldots, n\}, j \in\{1, \ldots, m\}
$$

(c) The VRPB may be modelled by setting $\gamma=1$ in constraint (13).

(d) The VRPDDP may be modelled as a VRPMDP by creating two fictitious customers for each genuine customer, one purely linehaul, one purely backhaul. Unfortunately this means that the problem is harder to solve as the number of customers is doubled. This VRPMDP may, in turn, be modelled as a VRPSDP.

(e) Adding (13) to the above yields a model for the VRPRMDDP.

This is a concise and efficient formulation, based on the concept of commodity flow. This way, we avoid the use of three-index variables. It is an improvement of an earlier formulation for the VRPRMDP by Nagy, Wassan and Salhi (2013) - while it contains more variables, it requires fewer inequalities.

We give a small illustration of solving small instances using this formulation with commercial software. Two datasets will be used, one for the case of single demands (VRPB, VRPMDP and VRPRMDP) and one for the case of combined demands (VRPSDP, VRPDDP and VRPRMDDP). All of them are adaptations of the well-known Christofides-Eilon VRP instances, namely CE22, CE23, CE30 and CE33, see Christofides and Eilon (1969). The number of customers is one less than the number of the instance, i.e., they consist of 21, 22, 29 and 32 customers. Coordinates and maximum capacities are unchanged. For the case of single demands, we create three instances for each VRP instance by declaring very second, every third or every fifth customer a backhaul. This means that for these customers we let $b_{i}=q_{i}$ and then let $q_{i}=0$ while for the remainder of customers we have $b_{i}=0$. The resulting instances are called CE22_2, CE22_3, CE22_5, etc. For the case of combined demands, we create just one instance for each VRP instance as follows. Let the delivery demand $q_{i}$ of each customer remain the same as its original demand in the VRP instance. Let the pickup of the first customer be $b_{1}=q_{n}$ and the pickup of the any other customer $i$ be $b_{i}=q_{i-1}$. The resulting instances are referred to as CE22P, CE23P, CE30P and CE33P.

These instances were solved using IBM ILOG CPLEX (version 12.5). Results for single demands are given in Table 1, results for combined demands are given in Table 2. For the sake of consistency, we did not use constraint (12) in any of the versions. (Traditionally a fixed fleet restriction is used by VRPB researchers, see Wassan (2007).) For both the VRPRMDP and the VRPRMDDP, a $\gamma$ value of $25 \%$ was used. We allowed a maximum running time of one day (= 86400 seconds); if the optimum was not found within this time, we report lower and upper bounds found. (This has only occurred for the VRPDDP and VRPRMDDP, as the number of customers had to be doubled in these versions.) 
Table 1. Results for Single Demands

\begin{tabular}{|l|c|c|c|c|c|c|c|c|c|}
\hline version & \multicolumn{3}{|c|}{ VRPB } & \multicolumn{3}{c|}{ VRPMDP } & \multicolumn{3}{c|}{ VRPRMDP } \\
\hline instance & $z$ & $k$ & $t$ & $z$ & $k$ & $t$ & $z$ & $k$ & $t$ \\
\hline CE22_2 & 371 & 3 & 1 & 324 & 3 & 7 & 326 & 3 & 5 \\
\hline CE22_3 & 366 & 3 & 1 & 341 & 3 & 1 & 348 & 3 & 2 \\
\hline CE22_5 & 375 & 3 & 1 & 341 & 3 & 2 & 341 & 3 & 2 \\
\hline CE23_2 & 670 & 3 & 1 & 526 & 2 & 8 & 526 & 2 & 244 \\
\hline CE23_3 & 640 & 3 & 1 & 526 & 2 & 13 & 533 & 2 & 45 \\
\hline CE23_5 & 623 & 2 & 1 & 514 & 2 & 315 & 514 & 2 & 388 \\
\hline CE30_2 & 501 & 3 & 5 & 417 & 2 & 9 & 432 & 3 & 4012 \\
\hline CE30_3 & 537 & 3 & 56 & 475 & 3 & 1362 & 475 & 3 & 1344 \\
\hline CE30_5 & 513 & 3 & 11 & 475 & 3 & 1505 & 475 & 3 & 1609 \\
\hline CE33_2 & 738 & 3 & 173 & 681 & 3 & 2001 & 694 & 3 & 7513 \\
\hline CE33_3 & 750 & 3 & 209 & 680 & 3 & 2930 & 681 & 3 & 29876 \\
\hline CE33_5 & 736 & 3 & 135 & 686 & 3 & 11572 & 686 & 3 & 10523 \\
\hline
\end{tabular}

$z$ : total route length, $k$ : number of vehicles, $t$ : computing time (in seconds)

Table 2. Results for Combined Demands

\begin{tabular}{|l|c|c|c|c|c|c|c|c|c|}
\hline \multicolumn{1}{|c|}{ version } & \multicolumn{3}{|c|}{ VRPSDP } & \multicolumn{3}{c|}{ VRPDDP } & \multicolumn{3}{c|}{ VRPRMDDP } \\
\hline instance & $z$ & $k$ & $t$ & $z$ & $k$ & $t$ & $z$ & $k$ & $t$ \\
\hline CE22P & 394 & 5 & 33 & 385 & 4 & 745 & {$[385,423]$} & 86400 \\
\hline CE23P & 597 & 3 & 32 & 571 & 3 & 643 & 571 & 3 & 73 \\
\hline CE30P & 545 & 4 & 32451 & {$[452,545]$} & 86400 & {$[457,562]$} & 86400 \\
\hline CE33P & 844 & 4 & 3614 & {$[774,842]$} & 86400 & {$[797,961]$} & 86400 \\
\hline
\end{tabular}

$z$ : total route length [or bounds], $k$ : number of vehicles, $t$ : computing time (in seconds)

For single demands, computing times vary from one second to eight hours. Thus, this is a more efficient formulation than that of Nagy, Wassan and Salhi (2013). Moreover, we have found the optimal solution for CE33_2/VRPMDP and CE33_2/VRPRMDP; the previous formulation did not lead to optima. Of course, this is a general formulation for the VRPDP; for the particular case of VRPB more efficient formulations exist and it can be solved to optimality for hundreds of customers, see Toth and Vigo (2002).

For combined demands, the situation depends on if the customer numbers need to be doubled or not. In the latter case, for the VRPSDP, computing times vary from half a minute to nine hours. In the former case (VRPDDP/VRPRMDDP), CPLEX could not find the optimum for several instances within the limit of one day. We note that Nagy, Wassan, Speranza and Archetti (2012) found the optimum for CE30P/VRPDDP after several days and additional inequalities. However, they have not considered the VRPRMDDP. In fact, the results given here are the first exact experiments on the VRPRMDDP, although Hoff and Løkketangen (2006) solved some small TSPRMDDP instances to optimality.

In the following, we make some observations on our experimental results, bearing in mind that this is a small experiment with just a few instances.

- For any instance, $z$ (VRPMDP) $\leq z($ VRPRMDP) $\leq z$ (VRPB).

- The average improvement achieved by allowing deliveries and pickups to occur in any order compared to the VRPB assumption is $12 \%$.

- By allowing deliveries and pickups to be onboard together subject to a $25 \%$ free space restriction we have, on average, an $11 \%$ better solution than the VRPB - nearly the 
same improvement as the VRPMDP model yet not having the problem of load shuffling.

- For any instance, $z$ (VRPDDP) $\leq z$ (VRPSDP).

- The average improvement achieved by allowing two visits to customers compared to the VRPSDP assumption is at least $2 \%$.

- There is not sufficient data to demonstrate the relationship between $z$ (VRPDDP) and $z$ (VRPRMDDP); note there is no relationship between $z$ (VRPSDP) and $z$ (VRPRMDDP).

- Having looked at the VRPDDP routes found for CE22P and CE23P, in both cases only two customers (out of 21-22) were split (served twice).

Further experimentation, additional valid inequalities and development of problemspecific exact solution methods should bring valuable insights to understanding these VRPDP versions.

\section{Metaheuristics Advances for the VRPDP Models}

While the VRPDP was proposed already in 1988 (Casco, Golden and Wasil (1988)), metaheuristic solution methods for this problem only appeared in this century. The most popular metaheuristic is without doubt tabu search, see Glover and Laguna (1997), but several other methodologies were proposed. In the following, we look at these methods for both traditional and novel VRPDP versions.

Osman and Wassan (2002) were the first to develop a tabu search meta-heuristic approach for the VRPB. In particular, they championed a very effective version of tabu search called reactive tabu search (RTS). They developed two route construction methods saving-insertion and saving-assignment to generate initial solution for their reactive tabu search algorithm to solve this problem. Their RTS approach uses a new diversification strategy that is different to the one originally proposed by Battiti and Tecchiolli (1994). Subsequently, RTS was used to solve nearly all versions of the VRPDP. The original RTS method was further improved and extended for the VRPSDP by Wassan, Nagy and Ahmadi (2008). This then was adapted for the VRPMDP by Wassan, Wassan and Nagy (2008). Initial solutions in both works were produced using a modified sweep method and their RTS phase relies on a number of neighbourhood routines, i.e., shift, swap, reverse. An RTS algorithm was used to solve the VRPRMDP by Nagy, Wassan and Salhi (2013), who carried out a detailed study of the effect of the free space parameter on the solution quality. Nagy, Wassan, Speranza and Archetti (2013) solved the VRPDDP and investigated (a) the types of instances where there are large cost differences between the VRPSDP and the VRPDDP models, (b) the characteristics of customers that are served twice in the VRPDDP, and (c) the shapes of VRPDDP routes.

There are also a number of classical (non-reactive) tabu search algorithms. The method of Brandão (2006) starts from a pseudo-lower bound to solve the VRPB. Tang and Galvão (2006) developed a tabu search algorithm for the VRPSDP that uses short- and long-term memory. Their initial solution methodologies designed use a number of methodologies, i.e., tour partitioning, sweep, and extensions of the TSP-based heuristics proposed in the literature. The tabu search neighbourhood moves are generated using insert, exchange, crossover and 2-Opt. Hoff and Løkketangen (2006) and Hoff, Gribkovskaia, Laporte and Løkketangen (2009) use tabu search based on a 2-opt neighbourhood to solve the VRPRMDDP, investigating different values of the free space and comparing lasso solutions against not having a fixed route shape. Chen and Wu (2006) also use tabu search for the VRPSDP that uses neighbourhood moves generated by 2-exchange, swap, shift, 2opt and Or-opt, however they generate initial solution by an insertion method, which relies on both distance- and load-based criteria. Bianchessi and Righini (2007) also investigate the VRPSDP. Their tabu search algorithm uses a combination of various arcexchange (cross involving two or three routes) and node-exchange (relocate, exchange) 
neighbourhoods. Two separate tabu lists are used (one for arc-based and one for nodebased neighbourhoods). Gribkovskaia, Halskau, Laporte and Vlček (2007) presented a tabu search metaheuristic for the VRPDDP based on splitting and merging customers. The main focus of this paper is on route shapes. Gribkovskaia, Laporte and Shyshou (2008) extend the above methods to the case where some pickups may be omitted (for a penalty).

Finally, tabu search may be even more effective when combined with other metaheuristics. Crispim and Brandão (2005) solved both the VRPSDP and the VRPMDP using a hybrid tabu search and variable neighbourhood algorithm that uses insert and swap routines, the initial solution is generated using the sweep method. Wassan (2007) developed a hybrid metaheuristic method that intelligently combines the features of adaptive memory programming with their previously developed RTS methodology in Osman and Wassan (2002). This approach proved faster and more efficient compared to Osman and Wassan (2002) in producing better quality solutions.

The second most popular VRPDP methodology seems to be ant colony optimisation. Wade and Salhi (2003) developed some enhancements on the standard ant colony approach for the VRPMDP such as a look-ahead based visibility, a site-dependent candidate list and efficient trail updating strategies, to tackle the deliveries and pickups in an efficient way. A recent improvement of this method is given by Wassan, Salhi, Nagy, Wassan and Wade (2013). Reimann and Ulrich (2006) designed an ant colony metaheuristic for the VRPRMDP.

Finally, we mention the adaptive large neighbourhood search algorithm of Ropke and Pisinger (2006). This can solve the VRPB, the VRPMDP and the VRPSDP, including time windows, hence the authors call it a unified heuristic. Very competitive results have been obtained. Tütüncü, Carreto and Baker (2009) used a GRASP metaheuristic to solve the VRPRMDP.

Although great advances were made in the past decade on metaheuristics for the VRPDP, there are plenty of promising ways research may move ahead. In our opinion, heuristics methods that traverse infeasible solutions (such as strategic oscillation) should be very suitable for the VRPDP, since its main issue is about checking feasibility. For strategic oscillation, see Kelly, Golden and Assad (1993), Nagy (1996) and Gallego, Laguna, Martí and Duarte (2013). Crispim and Brandão (2005) and Bianchessi and Righini (2007) allow infeasible intermediate solutions when solving the VRPSDP. Another possible research avenue is hybrid metaheuristics, in particular matheuristics. These exact-heuristic hybrids are very much in vogue nowadays and have been applied successfully to a number of VRP variants (Archetti, Speranza and Savelsbergh (2008), Maniezzo, Stützle and Voß (2009), Doerner and Schmid (2010), Salhi, Wassan and Hajarat (2013)), although they have not yet been applied to any VRPDP versions.

\section{Conclusions}

In this paper we have studied a very interesting and practical logistical problem which has attracted a lot of attention in the literature. The research has shown that the backhauling aspect of the vehicle routing problem is an interesting and practical application in the reverse logistics. Many businesses do backhauling in their fleet logistical operations. Here we have investigated a class of the classical vehicle routing problem variants, called VRPs with deliveries and pickups. We presented various problem versions including traditional and new ones with clear differences in terms of their practice and modelling issues especially focusing on the assumptions generally made in the literature and on the benefits of not making too restrictive assumptions. We have also highlighted the research issues on novel problem classes that are rarely discussed in the literature. As the reader can see, the Vehicle Routing Problem with Deliveries and 
Pickups has lately proved to be an exciting topic for researchers. Nevertheless, there is some confusion about problem classes - we hope to have contributed here to mitigate this.

Moreover we have presented an ILP formulation for the VRPPD and shown how this can be modified to model the other problem classes. We anticipate this would not only provide new researchers a point of start but help them to produce new extensions to bring this problem even closer to the reality. We also discussed various solution methodologies developed to solve those models and what more is needed in this important research area. However, there is still a lot to explore, and we hope young researchers will be excited with this topic and contribute to developing it further in order to bridge the gap between the academia and the industry even closer.

\section{References}

[1] S. Anily, "The Vehicle Routing Problem with Delivery and Back-haul Options", Naval Research Logistics, vol. 43, (1996), pp. 415-434.

[2] C. Archetti and M. G. Speranza, "Vehicle Routing Problems with Split Deliveries", International Transactions in Operational Research, vol. 39, (2012), pp. 3-22.

[3] C. Archetti, M. G. Speranza and M. W. P. Savelsbergh, "An Optimization-based Heuristic for the Split Delivery Vehicle Routing Problem”, Transportation Science, vol. 42, (2008), pp. 22-31.

[4] M. Battarra, G. Erdoğan, G. Laporte and D. Vigo, "The Traveling Salesman Problem with Pickups", Deliveries, and Handling Costs, Transportation Science, vol. 44, (2010), pp. 383-399.

[5] R. Battiti and G. Tecchiolli, "The Reactive Tabu Search", ORSA Journal on Computing, vol. 6, (1994), pp. 126-140.

[6] G. Berbeglia, G.-F. Cordeau, I. Gribkovskaia and G. Laporte, "Static Pickup and Delivery Problems: A Classification Scheme and Survey, TOP, vol. 15, (2007), pp. 1-31.

[7] N. Bianchessi and G. Righini, "Heuristic Algorithms for the Vehicle Routing Problem with Simultaneous Pick-up and Delivery”, Computers \& Operations Research, vol. 34, no. 2, (2007), pp. 578-594.

[8] J. Brandão, "A New Tabu Search Algorithm for the Vehicle Routing Problem with Backhauls", European Journal of Operational Research, vol. 173, (2006), pp. 540-555.

[9] D. O. Casco, B. L. Golden and E. A. Wasil, "Vehicle Routing with Backhauls: Models, Algorithms and Case Studies", B. L., Golden \& A. A. Assad ( Ed.), Vehicle Routing: Methods and Studies, Elsevier, (1988), pp. 127-147.

[10] J.-F. Chen and T.-H. Wu, "Vehicle Routing Problem with Simultaneous Deliveries and Pickups", Journal of the Operational Research Society, vol. 57, (2006), pp. 579-587.

[11] N. Christofides and S. Eilon, "An Algorithm for the Vehicle Routing Dispatching Problem", Operational Research Quarterly, vol. 20, (1969), pp. 309-318.

[12] J. Crispim and J. Brandão, "Metaheuristics Applied to Mixed and Simultaneous Extensions of Vehicle Routing Problems with Backhauls", Journal of the Operational Research Society, vol. 56, (2005), pp. 1296-1302.

[13] K. Doerner and V. Schmid, "Matheuristics for Rich Vehicle Routing Problems", M. Blesa, C. Blum, G. Raidl, A. Roli, M. Sampels (Ed.), Hybrid Metaheuristics Lecture Notes in Computer Science, Springer, Berlin/Heidelberg, vol. 6373, (2010), pp. 206-221.

[14] M. Gallego, M. Laguna, R. Martí and A. Duarte, "Tabu Search with Strategic Oscillation for the Maximally Diverse Grouping Problem", Journal of the Operational Research Society, vol. 64, (2013), pp. 724-734.

[15] F. Glover and M. Laguna, Tabu Search, Boston: Kluwer, (1997).

[16] I. Gribkovskaia, Ø. Halskau, G. Laporte and M. Vlček, "General Solutions to the Single Vehicle Routing Problem with Pickups and Deliveries", European Journal of Operational Research, vol. 180, (2007), pp. 568-584.

[17] I. Gribkovskaia, G. Laporte and A. Shyshou, "The Single Vehicle Routing Problem with Deliveries and Selective Pickups", Computers \& Operations Research, vol. 35, (2008), pp. 2908-2924.

[18] Ø. Halskau, I. Gribkovskaia and K. N. B. Myklebost, "Models for Pick-up and Deliveries from Depots with Lasso Solutions", G. Stefansson \& B. Tilanus (Ed.), Proceedings of the 13th Annual Conference on Logistics Research NOFOMA 2001, Chalmers University of Technology, Göteborg, (2001), pp. 279293.

[19] A. Hoff and A. Løkketangen, "Creating Lasso-solutions for the Traveling Salesman Problem with Pickup and Delivery by Tabu Search”, Central European Journal of Operations Research, vol. 14, (2006), pp. 125-140.

[20] A. Hoff, I. Gribkovskaia, G. Laporte and A. Løkketangen, "Lasso Solution Strategies for the Vehicle Routing Problem with Pickups and Deliveries", European Journal of Operational Research, vol. 192, (2009), pp. 755-766. 
[21] M. Iori and S. Martello, "Routing Problems with Loading Constraints", TOP, vol. 18, (2010), pp. 4-27.

[22] J. P. Kelly, B. L. Golden and A. A. Assad, "Large-scale Controlled Rounding using Tabu Search with Strategic Oscillation", Annals of Operations Research, vol. 41, no. 2, (1993), pp. 69-84.

[23] V. Maniezzo, T. Stützle and S. Voß, "Matheuristics. Hybridizing Metaheuristics and Mathematical Programming", Annals of Information Systems, Springer, Heidelberg, vol. 10, (2009).

[24] S. Mitra, "An Algorithm for the Generalized Vehicle Routing Problem with Backhauling", Asia-Pacific Journal of Operational Research, vol. 22, (2005), pp. 153-169.

[25] S. Mitra, "A Parallel Clustering Technique for the Vehicle Routing Problem with Split Deliveries and Pickups", Journal of the Operational Research Society, vol. 59, (2008), pp. 1532-1546.

[26] G. Mosheiov, "The Travelling Salesman Problem with Pick-up and Delivery", European Journal of Operational Research, vol. 79, (1994), pp. 299-310.

[27] G. Nagy, "Heuristic Methods for the Many-to-Many Location-Routing Problem", PhD thesis, School of Mathematics and Statistics, The University of Birmingham, Birmingham, (1996).

[28] G. Nagy and S. Salhi, "Heuristic Algorithms for Single and Multiple Depot Vehicle Routing Problems with Pickups and Deliveries", European Journal of Operational Research, vol. 162, (2005), pp. 126-141.

[29] G. Nagy, N. A. Wassan and S. Salhi, "The Vehicle Routing Problem with Restricted Mixing of Deliveries and Pickups", Journal of Scheduling, vol. 16, (2013), pp. 199-213.

[30] G. Nagy, N. A. Wassan, M. G. Speranza and C. Archetti, "The Vehicle Routing Problem with Divisible Deliveries and Pickups", Transportation Science (to appear), (2013).

[31] S. N. Parragh, K. F. Doerner and R. F. Hartl, "A Survey on Pickup and Delivery Problems", Part I: Transportation between Customers and Depot, Journal für Betriebswirtschaft, vol. 58, (2008), pp. 21-51.

[32] R. Reimann and H. Ulrich, "Comparing Backhauling Strategies in Vehicle Routing using Ant Colony Optimization”, Central European Journal of Operations Research, vol. 14, (2006), pp. 105-123.

[33] S. Ropke and D. Pisinger, "A Unified Heuristic for a Large Class of Vehicle Routing Problems with Backhauls", European Journal of Operational Research, vol. 171, (2006), pp. 750-775.

[34] S. Salhi and G. Nagy, "A Cluster Insertion Heuristic for Single and Multiple Depot Vehicle Routing Problems with Backhauling", Journal of the Operational Research Society, vol. 50, (1999), pp. 10341042.

[35] S. Salhi, N. Wassan and M. Hajarat, "The Fleet Size and Mix Vehicle Routing Problem with Backhauls: Formulation and Set Partitioning-based Heuristics", Transportation Research Part E, vol. 56, (2013), pp. 22-35.

[36] F. A. Tang and R. D. Galvão, "A Tabu Search Algorithm for the Vehicle Routing Problem with Simultaneous Pickup and Delivery Service", Computers \& Operations Research, vol. 33, (2006), pp. 595-619.

[37] P. Toth and D. Vigo, “The Vehicle Routing Problem”, Philadelphia: Society for Industrial and Applied Mathematics, (2002).

[38] G. Y. Tütüncü, C. A. C. Carreto and B. M. Baker, "A Visual Interactive Approach to Classical and Mixed Vehicle Routing Problems with Backhauls", Omega, vol. 37, (2009), pp. 138-154.

[39] A. C. Wade and S. Salhi, "An Investigation into a New Class of Vehicle Routing Problem with Backhauls”, Omega, vol. 30, (2002), pp. 479-487.

[40] A. C. Wade and S. Salhi, "An Ant System Algorithm for the Mixed Vehicle Routing Problem with Backhauls", Metaheuristics: Computer Decision-Making, M. G. Resende and J. P. de Sousa (eds.), Kluwer, New York, (2003), pp. 699-719.

[41] N. A. Wassan and I. H. Osman, "Tabu Search Variants for the Mix Fleet Vehicle Routing Problem", Journal of the Operational Research Society, vol. 53, (2002), pp. 768-782.

[42] N. A. Wassan, G. Nagy and S. Ahmadi, "A Heuristic Method for the Vehicle Routing Problem with Mixed Deliveries and Pickups", Journal of Scheduling, vol. 11, (2008), pp. 149-161.

[43] N. Wassan, "Reactive Tabu Adaptive Memory Programming Search for the Vehicle Routing Problem with Backhauls", Journal of the Operational Research Society, vol. 58, (2007), pp. 1630-1641. 


\section{Authors}
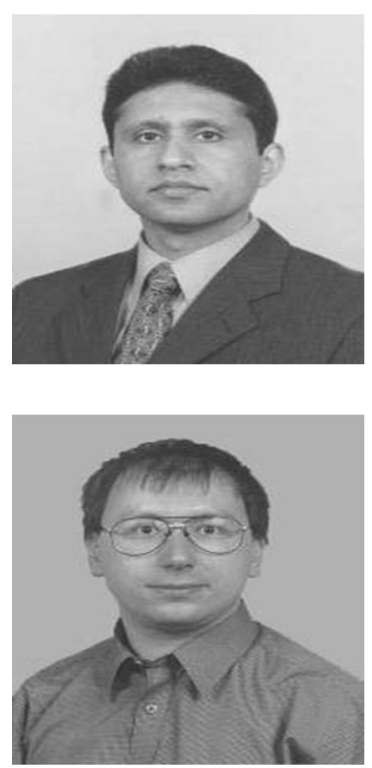

Dr Niaz Wassan received his PhD from the University of Kent. He is currently a Senior Lecturer in Management Science at the University of Kent. His research interests include logistics, vehicle routing heuristics and meta-heuristics.

Dr Gabor Nagy received his PhD from the University of Birmingham, where he was supervised by Professor Said Salhi. He is currently a Senior Lecturer in Management Science at the University of Kent. His research interests include lacation theory, vehicle routing and heuristics. 